\title{
Thermal and fast neutron dosimetry using artificial single crystal diamond detectors
}

\author{
M. Angelone ${ }^{\mathrm{a}, *}$, M. Pillon $^{\mathrm{a}}$, G. Prestopino $^{\mathrm{a}}$, Marco Marinelli ${ }^{\mathrm{b}}$, E. Milani $^{\mathrm{b}}$, C. Verona $^{\mathrm{b}}$, G. Verona-Rinati $^{\mathrm{b}}$,

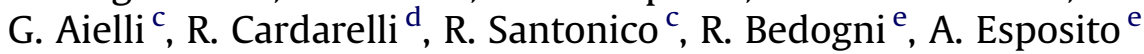 \\ a Associazione EURATOM-ENEA sulla Fusione, ENEA C.R. Frascati, Via E. Fermi, 45, I-00044 Frascati, Rome, Italy \\ b Dipartimento di Ingegneria Meccanica dell'Università degli Studi “Tor Vergata", Via del Politecnico, 1, 00100 Rome, Italy \\ 'Dipartimento di Fisica dell'Università degli Studi "Tor Vergata”, Rome, Italy \\ disituto Nazionale di Fisica Nucleare, sez. "Tor Vergata", Rome, Italy \\ e Isituto Nazionale di Fisica Nucleare, Laboratori Nazionali di Frascati, via E. Fermi, 40, 00044 Frascati, Italy
}

\section{A R T I C L E I N F O}

\section{Article history:}

Received 26 October 2010

Received in revised form

15 June 2011

Accepted 16 June 2011

\section{Keywords:}

Thermal neutrons

Fast neutrons

Neutron dosimetry

Diamond detectors

Response functions

Ambient dose equivalent

\begin{abstract}
A B S T R A C T
In this work we propose the artificial Single Crystal Diamond (SCD) detector covered with a thin layer $(0.5 \mu \mathrm{m} / 4 \mu \mathrm{m})$ of ${ }^{6} \mathrm{LiF}$ as a simultaneous thermal and fast neutron fluence monitor. Some interesting properties of the diamond response versus the neutron energy are evidenced thanks to Monte Carlo simulation using the MCNPX code which allows to propose the diamond detector also as an ambient dose equivalent $\left(\mathrm{H}^{*}(10)\right)$ monitor (REM counter).
\end{abstract}

() 2011 Elsevier Ltd. All rights reserved.

\section{Introduction}

The development of a reliable procedure to produce artificial single crystal diamond (SCD) has boosted the interest for diamond detectors because of their many outstanding properties such as high energy resolution, high carrier mobility and high resistivity which make the SCD a very interesting detector for radiation detection.

Applications of artificial diamond detectors are reported for neutrons measurements in fusion tokamaks, charged particle detection as well as for soft X-rays and UV (Angelone et al., accepted for publication; Almaviva et al., 2009). These applications are intended to neutron counting and spectrometry, by including an attempt to use the diamond as an X-ray dosemeter in medical applications (Almaviva et al., 2009b). Up to now there are just a few papers reporting the use of diamonds in neutron dosimetry. Among them, an SCD detector covered with a very thin Boron layer $(<1 \mu \mathrm{m})$ was proposed for boron neutron capture therapy (BNCT) (Almaviva et al., 2010a).

\footnotetext{
* Corresponding author. Tel.: +39 (0) 694005518; fax: +39 (0) 694005314

E-mail address: maurizio.angelone@enea.it (M. Angelone).
}

Indeed, diamonds are attractive for neutron dosimetry since their atomic number $(Z=6)$ is comparable with that of tissues. The low $Z$ value also ensures low sensitivity to gammas and this can help in mixed neutron-gamma $(\mathrm{n}-\gamma)$ radiation fields.

Applications for high energy neutron detection or for area monitoring around particle accelerators have not yet been investigated. However, the recent development of a new type of amplifier (Angelone et al., accepted for publication, sect. 3 ) that allows to locate the SCD detector far away from the amplifier (up to $100 \mathrm{~m}$ ) opens the way to use the SCD detectors in harsh environments such as those expected around accelerators or tokamaks.

In this work, we studied an artificial SCD detector coupled to a ${ }^{6} \mathrm{LiF}$ converter as a simultaneous thermal and fast neutron fluence monitor. Some interesting properties of the diamond response versus the neutron energy are also evidenced thanks to Monte Carlo simulations performed with the MCNPX code. These simulations allowed to study the SCD detector both as a neutron counter and as a dosemeter.

\section{The SCD detector}

Single crystal diamond (SCD) detectors produced by chemical vapor deposition technique (CVD) represent the state-of-the-art of 
present technology (Marinelli et al., 2006). Commercially available SCD detectors are usually fabricated with two metal ( $\mathrm{Au}, \mathrm{Al}, \mathrm{Pt}, \mathrm{Cr}$ ) contacts or can be provided with a special designed contact (Galbiati et al., 2009) made of a metal alloy and carbon-like material.

The SCD shows high energy resolution $(<0.5 \%)$ but for long lasting measurements the polarization could be a problem since the resolution is dramatically worsened. A solution to these polarization effects was found at the Tor Vergata University (Rome, Italy) by producing detectors with a layered structure (Marinelli et al., 2006). These detectors are produced by a two step process. In the first step a thin layer $(10-15 \mu \mathrm{m})$ of SCD diamond highly doped with boron (boron concentration about $5 \times 10^{-19} \mathrm{at}^{*} \mathrm{~cm}^{-3}$ ) is grown on top of the diamond substrate. The latter is a commercially available SCD of high pressure high temperature (HPHT) type and thus of poor quality. In the second step a high purity, nominally intrinsic layer of diamond is grown (electrically active acceptor-like defect concentration of about $10^{14} \mathrm{~cm}^{-3}$ ). Its thickness varies from a few microns up to $200 / 300 \mu \mathrm{m}$, depending on the application. This intrinsic layer acts as active medium while the doped layer is the backing contact. A second metallic contact $(\mathrm{Al}, \mathrm{Cr}, \mathrm{Au}, \mathrm{Pt})$ is then deposited on the top of the intrinsic layer. The main advantages of this layered detector are the absence of polarization effects and the presence of an intrinsic internal voltage of about $1 \mathrm{~V}$ (Almaviva et al., 2010b) which in some applications (e.g. UV, soft X-ray, intense radiation fields) allows for the use of the detector without any external bias (H.V.).

A further step was the deposition of a thin layer $(0.5 \mu \mathrm{m}-4 \mu \mathrm{m})$ of ${ }^{6} \mathrm{LiF}$ on top of the metallic contact. ${ }^{6} \mathrm{Li}$ is highly sensitive to thermal neutrons via the ${ }^{6} \mathrm{Li}(\mathrm{n}, \alpha) \mathrm{T}$ reaction. The high energy resolution allows the $T$ and alpha peaks to be separated in pulse height spectrum (PHS). Therefore, after calibration in a reference thermal field, they can be used to measure the thermal fluence and hence the thermal neutron dose. The Lithium Diamond (LiDia) detector can be used to detect simultaneously thermal and fast neutrons (Marinelli et al., 2006).

\section{Signal detection and electronics}

Diamond produces signals proportional to the energy of the ionizing particle. However, its band gap $(5.3 \mathrm{eV})$ leads to the generation of electric signals of low amplitudes (tens $\mu \mathrm{V}$ ). In contrast, the electric signals are very fast (hundreds of ps) for its high carriers mobility. To handle and transport far away (tens of meters) this electrical signal is not an easy task if PHS has to be recorded in harsh environments since commercial charge preamplifiers posses a slow dynamics and are sensitive to cable impedance and to radiation. High frequency, low attenuation, super-screened cables are suitable to transport the SCD signal far away (up to $100 \mathrm{~m}$ ) provided that their impedance matches with the input impedance of the charge pre-amplifier.

A Matching Sensitive Charge Amplifier (MSCA) of a new concept was developed in collaboration with the Dept. of Physics of the Tor Vergata Univiversity in Rome and Istituto Nazionale di Fisica Nucleare (INFN) (Tor Vergata section) (Angelone et al., accepted for publication). A very high performance two stage front-end electronics was developed with extremely high lock-in capability for fast signals. The MSCA is able to read, amplify and stretch (up to $100 \mathrm{~ns}$ ) the small and ultra fast signal produced in the diamond detector. The amplifier gives an output which is linear vs. the injected charge in terms of both signal amplitude and area. A custom design and appropriate matching of the electronic components with diamond detector permitted to reach an unprecedent combination of speed and signal to noise ratio $(\mathrm{S} / \mathrm{N})$. Emphasis was given to the compensation of the negative

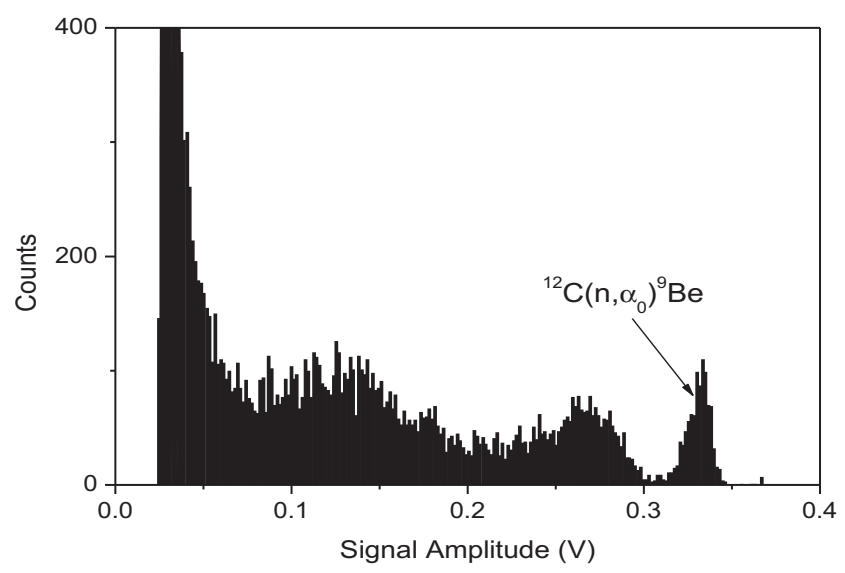

Fig. 1. PHS recorded with a digital oscilloscope using the new MSCA and an SCD detector irradiated at FNG with $14 \mathrm{MeV}$ neutrons.

undershoot which is essential to have a good response linearity even with a high repetition rate. The amplification process can be optimized by using a base-emitter configuration avoiding the collector configuration since this enlarges the working band which now is restricted to a few $\mathrm{MHz}$. This configuration "automatically" selects the frequencies to be amplified and thus reduces the noise level. The signal from the MSCA is then processed throughout a commercial fast digitizer for PHS recording (spectrometric mode), but the system can operate simultaneously in counting mode.

Tests were performed at the $14 \mathrm{MeV}$ Frascati Neutron Generator (FNG) (Martone et al., 1994) to measure the ${ }^{12} C\left(n, \alpha_{0}\right){ }^{9}$ Be reaction by using $40 \mathrm{~m}$ long cable. An example of PHS measured at FNG using the MSCA is reported in Fig. 1.

\section{Response of diamond to fast neutrons}

The first application of a natural diamond $\left(99 \%{ }^{12} \mathrm{C}\right.$ and $\left.1 \%{ }^{13} \mathrm{C}\right)$ as a neutron detector was addressed to $14 \mathrm{MeV}$ neutron spectrometry (Pillon et al., 1995). Table 1 lists the neutron reactions occurring on ${ }^{12} \mathrm{C}$ for neutron energy up to $150 \mathrm{MeV}$. Fig. 2 shows some of the most important neutron reaction cross sections on carbon up to $150 \mathrm{MeV}$.

For neutron energy $\left(E_{\mathrm{n}}\right)$ up to $20 \mathrm{MeV}$ the most important reaction for spectroscopy is ${ }^{12} \mathrm{C}\left(\mathrm{n}, \alpha_{0}\right){ }^{9} \mathrm{Be}(Q=5.701 \mathrm{MeV})$ in which the $\alpha$ and ${ }^{9} \mathrm{Be}$ particles with a total kinetic energy $T=E_{\mathrm{n}}-Q$ are produced. These charged particles stop inside the crystal with a very short range $\left(<30 \mu \mathrm{m}\right.$ for $\left.E_{\mathrm{n}}=20 \mathrm{MeV}\right)$ so releasing all their energy to the crystal where e-hole pairs are generated.

Recently the response functions of a commercial SCD detector $500 \mu \mathrm{m}$ in thickness where measured in the neutron energy range from 6 up to $20 \mathrm{MeV}$ at the Van der Graaf neutron generator

\section{Table 1}

Nuclear reactions in ${ }^{12} \mathrm{C}$.

\begin{tabular}{lcc}
\hline Reaction & Q-value $(\mathrm{keV})$ & Threshold $(\mathrm{keV})$ \\
\hline${ }^{12} \mathrm{C}(\mathrm{n}, \gamma){ }^{13} \mathrm{C}$ & 4946.31 & 0.0 \\
${ }^{12} \mathrm{C}(\mathrm{n}, \alpha){ }^{9} \mathrm{Be}$ & -5701.25 & 6180.60 \\
${ }^{12} \mathrm{C}\left(\mathrm{n}, \mathrm{n}^{\prime} 2 \alpha\right){ }^{4} \mathrm{He}$ & -7274.75 & 7886.40 \\
${ }^{12} \mathrm{C}\left(\mathrm{n}, \mathrm{n}^{\prime} \alpha\right){ }^{8} \mathrm{Be}$ & -7366.59 & 7985.96 \\
$\left.{ }^{12} \mathrm{C}(\mathrm{n}, 2 \alpha)\right)^{5} \mathrm{He}$ & -8164.7 & 8851.2 \\
${ }^{12} \mathrm{C}(\mathrm{n}, \mathrm{p}){ }^{12} \mathrm{~B}$ & -12586.55 & 13644.81 \\
${ }^{12} \mathrm{C}(\mathrm{n}, \mathrm{d}){ }^{11} \mathrm{~B}$ & -13732.33 & 14886.93 \\
${ }^{12} \mathrm{C}(\mathrm{n}, \mathrm{n} \cdot \mathrm{p}){ }^{11} \mathrm{~B}$ & -15956.90 & 17298.53 \\
${ }^{12} \mathrm{C}(\mathrm{n}, 2 \mathrm{n}){ }^{11} \mathrm{C}$ & -18.721 .66 & 20295.75 \\
${ }^{12} \mathrm{C}(\mathrm{n}, \mathrm{T}){ }^{10} \mathrm{~B}$ & -18929.22 & 20520.77 \\
\hline
\end{tabular}




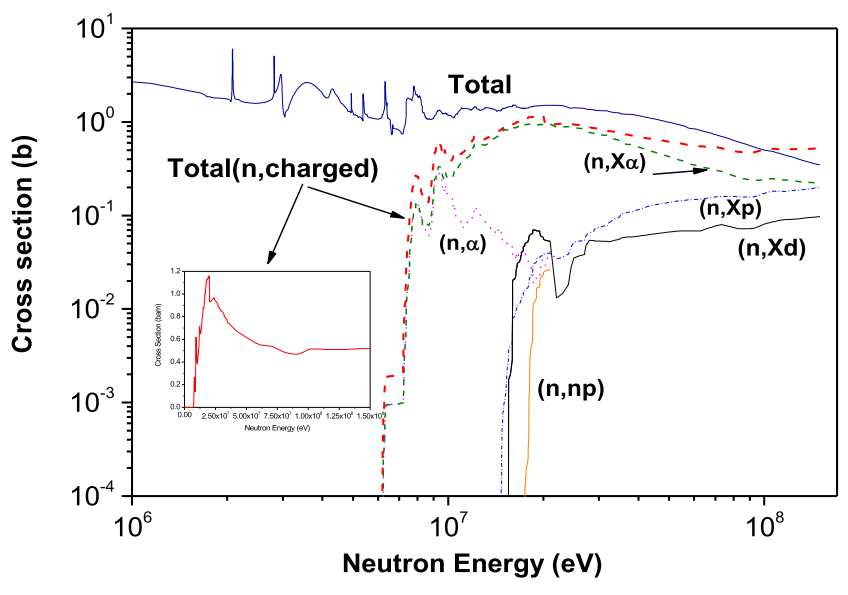

Fig. 2. Carbon cross section versus neutron energy.

available at JRC in Geel, as part of the EUFRAT program (http:// irmm.jrc.ec.europa.eu/activities/eufrat). Some of the measured responses (PHS) are shown in Fig. 3. These spectra were normalized to the total number of neutrons impinging on the detector, i.e. the product of the neutron fluence and the area of the detector. The sharp peaks produced by the ${ }^{12} \mathrm{C}\left(\mathrm{n}, \alpha_{0}\right){ }^{9} \mathrm{Be}$ reaction are visible, their area being proportional to the neutron fluence. The other peaks in the PHS can be attributed to other reactions occurring in ${ }^{12} \mathrm{C}$ or ${ }^{13} \mathrm{C}$ (depending on $E_{\mathrm{n}}$, Table 1 ). The peaks width (FWHM) is related to the energy spread of the incoming neutron beam and the intrinsic resolution of the SCD detector. The low energy continuous is mainly due to inelastic reactions in carbon.

\subsection{SCD as neutron dosimeter}

The area of the measured PHS yields the total response for the SCD and can be plotted vs. neutron energy. A proper energy threshold (Fig. 3) should be introduced in the PHS to cut the contribution of the electrical noise and gamma radiation. The experimental total response so obtained was normalized to the total neutron fluence and to the detector volume (Fig. 4).

The correlation of the response functions with dosimetric quantities was investigated in the present work. The response functions were calculated with a Monte Carlo transport code for an SCD detector exposed to monochromatic neutrons whose energy ranges from 7 up to $800 \mathrm{MeV}$. For each neutron energy the reaction

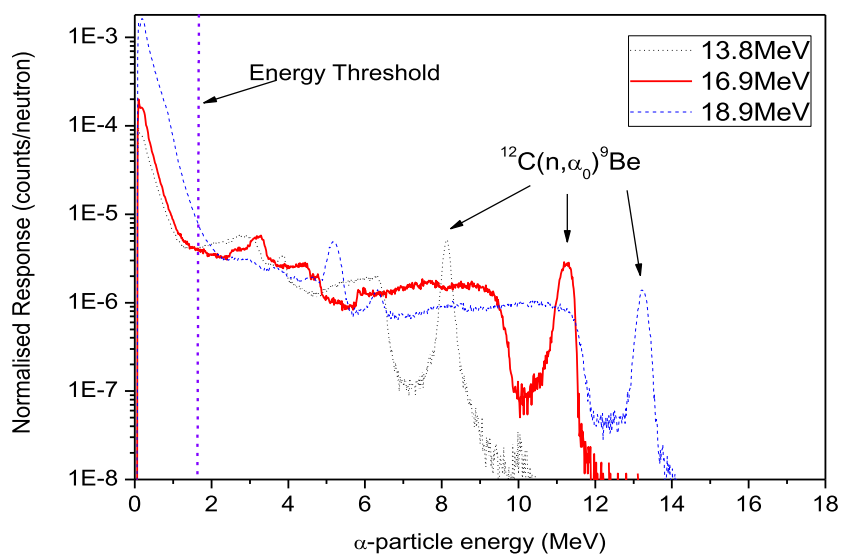

Fig. 3. PHS for the SCD detector measured at three different neutron beam energies using commercial pre-amplifier.

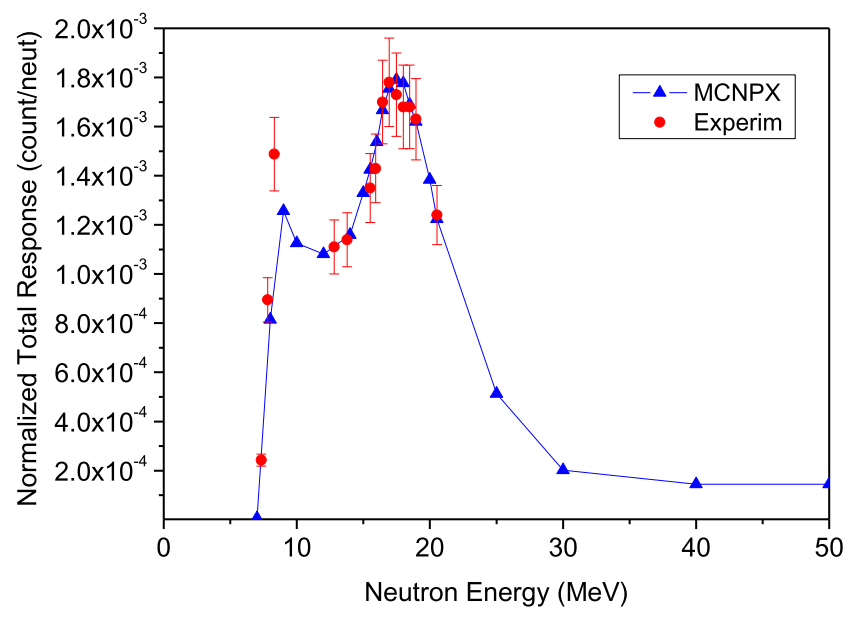

Fig. 4. Relative comparison between calculated total carbon responses using MCNPX2.5 and the total response (count/neut.) measured at IRMM-Geel in the neutron energy range $7-20 \mathrm{MeV}$.

rates for the most relevant nuclear reactions in carbon were computed with the MCNPX-2.5 code (Pelowitz, 2005). The reaction rate on carbon was calculated by folding the neutron fluence (tally 4, MCNPX) with the material cross sections from ENDF/B7 crosssection file. The sum of the calculated reaction rates gives the total detector response (TR). The calculated TR, after normalization to the detector volume, were compared with the experimental ones (up to $20 \mathrm{MeV}$ ) and the results are shown in Fig. 4. The agreement is good.

A new series of MCNPX simulations was performed for the LiDia detector ( $S C D$ covered with $3 \mu \mathrm{m}$ of ${ }^{6} \mathrm{LiF}$ ) placed at the center of two polyethylene Bonner Spheres $3^{\prime \prime}$ and $8^{\prime \prime}$ in diameter and two spheres $12^{\prime \prime}$ in diameter, one of which equipped with $1 \mathrm{~cm}$ lead shell. The LiDia detector allows for the simultaneous detection of thermal and fast neutrons, thus a spectrometer sensitive to both fast and thermal neutron energies could be designed. The results of the simulations are shown in Fig. 5.

Bonner spheres with ${ }^{6} \mathrm{LiF}$ detector are used since many years as neutron spectrometers and their response functions (both experimental and calculated) are available in the literature (Birattari et al., 2010). However ${ }^{6} \mathrm{LiF}$ is very sensitive to low energy neutrons but

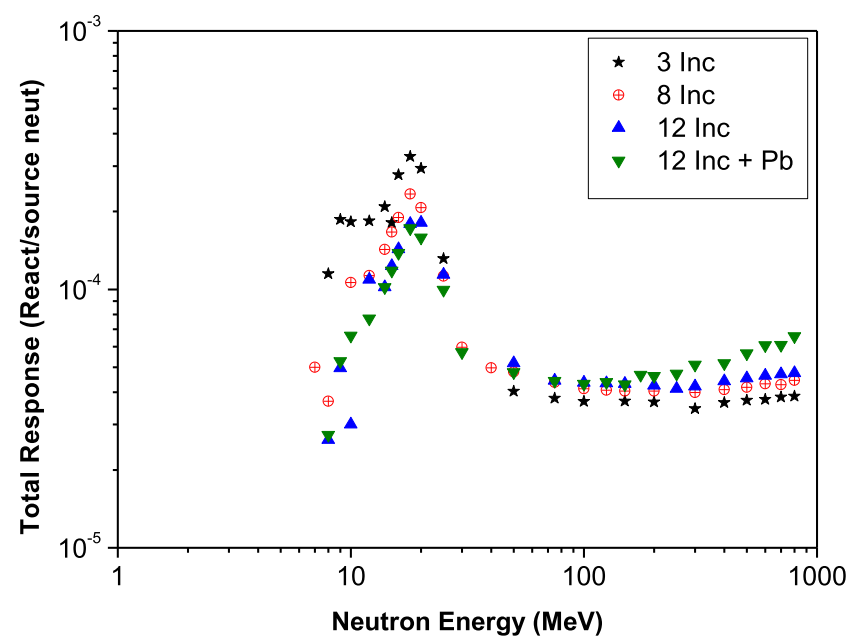

Fig. 5. Calculated MCNPX responses vs. neutron energy for SCD inside different Bonner spheres for neutron energy up to $800 \mathrm{MeV}$ (responses are reaction rates per source neutron). 


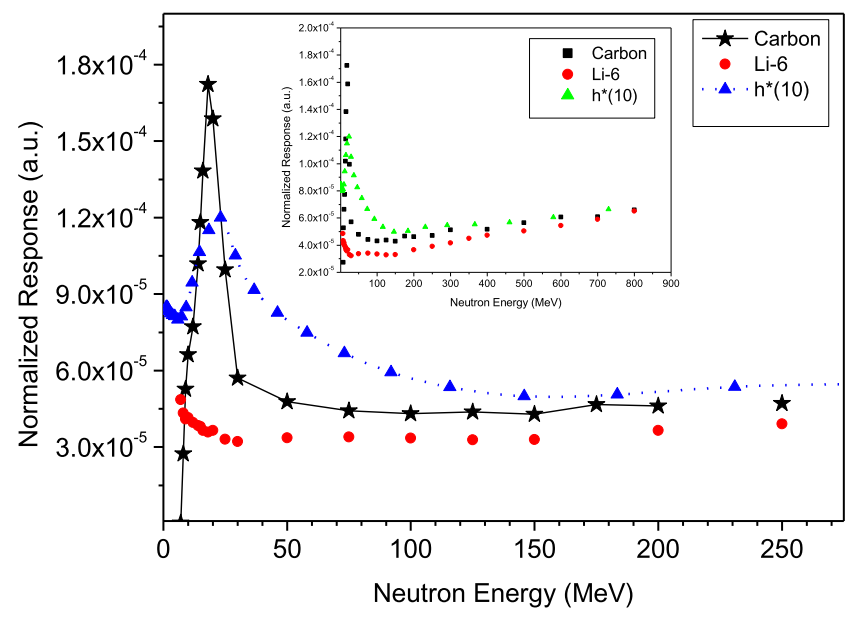

Fig. 6. Comparison between calculated total response in Carbon and $(n, T)$ response in ${ }^{6} \mathrm{Li}$ for LiDia located inside LINUS and the $h^{*}(10)$ coefficients from ICRP-74. This is a comparison among the behavior (versus neutron energy) of different quantities which are reported in a single vertical scale (a.u.) after arbitrary normalization.

scarcely sensitive to fast neutrons, so above $10 \mathrm{MeV}$ it cannot provides much information about the neutron spectrum. The same applies to other active detectors coupled to Bonner spheres (e.g. the He-3 counter).

In the recent years attempts were made to improve the response to high energy neutrons of Bonner spheres by using an He-3 proportional counter. A $12^{\prime \prime}$ sphere containing a lead shell $1 \mathrm{~cm}$ in thickness (LINUS detector, Birattari et al., 1990) has proven to extend the response to high energy neutrons (up to about $30 \mathrm{MeV}$ ). The LINUS has been proposed as REM counter since its response versus the neutron energy mimics the trend against neutron energy of the conversion coefficients of fluence to ambient dose equivalent for neutrons (ICRP-74 publication, 1997) (hereafter the ambient dose equivalent is labeled with $H^{*}(10)$, while the conversion coefficients with $\left.h^{*}(10)\right)$. However, between 10 and $50 \mathrm{MeV}$, the LINUS response shows a deviation, being not capable of reproducing the peak appearing in the $h^{*}(10)$ curve.

An SCD (with ${ }^{6} \mathrm{LiF}$ converter) was placed at the center of the LINUS. The response of the new survey meter simulated with MCNPX is compared with the $h^{*}(10)$ curve in Fig. 6 . The response shows a trend similar to the $h^{*}(10)$ curve even in the region between 10 and $50 \mathrm{MeV}$ since the carbon response shows a sharp peak (inset of Fig. 2) which decreases around 25-30 MeV. This is mainly due to the sharp decrease of the ${ }^{12} C\left(n, \alpha_{0}\right){ }^{9} B e$ reaction above $20 \mathrm{MeV}$. At higher neutron energies the two curves are parallel (inset of Fig. 6). This survey meter (LINUS + SCD) can be calibrated with monoenergetic neutrons (e.g. Fig. 4), this allows to propose the new detector as REM counter. Future applications are foreseen around the DAФNE accelerator at INFN-LNF (Italy). This new instrument will be irradiated in position already qualified by conventional Bonner spheres (Esposito et al., 2010).

\section{Discussion and conclusion}

The SCD detector has demonstrated to be applied as a thermal neutron counter when coupled to a LiF converter and as a survey meter when placed at the center of the LINUS moderator/attenuator. A draw-back is the low efficiency of the SCD detector (see Fig. 5) which depends upon its small volume. Larger volumes are difficult to be produced since SCD films grow on top of small monocrystalline diamond films of poor quality. A solution can be the use of high quality polycrystalline diamond films which can be produced by using silicon as growing substrate, so much larger volumes can be obtained. Polycrystalline diamonds do not show spectrometric performances but their use as a survey meter is very promising.

In this work, the artificial SCD detector coupled to a ${ }^{6} \mathrm{LiF}$ converter was proposed as a simultaneous thermal and fast neutron fluence monitor. Some interesting properties of the total diamond response against neutron energy were investigated with MCNPX simulations.

\section{References}

Almaviva, S., Marinelli, M., Milani, E., et al., 2009a. Extreme UV single crystal diamond Schottky photodiode in planar and transverse configuration. Diam. Relat. Mater. 19, 78-82.

Almaviva, S., Ciancaglioni, L., Concorti, R., et al., 2009b. Synthetic single crystal diamond dosimeters for Intensity Modulated Radiation Therapy applications. Nluc. Instrum. Meth. in Phys. Res. A 608, 191-197.

Almaviva, S., Marinelli, M., Milani, E., et al., 2010a. Improved performance in syntetic diamond neutron detectors: application to boron neutron capture therapy. Nucl. Instrum. Meth. Phys. Res. A 612, 580-582.

Almaviva, S., Marinelli, M., Milani, E., et al., 2010b. Chemical vapor deposition diamond based multilayered radiation detector: physical analysis of detection properties. J. Appl. Phys. 107, 014511.

Angelone, M., Aielli, G., Almaviva, s. et.al. Neutron spectroscopy by means of artificial diamond detectors using a remote read-out scheme. IEEE Trans. Nucl. Sci., accepted for publication.

Birattari, C., Ferrari, A., Nuccetelli, C., Pelliccioni, M., Rancati, T., Silari, M., 1990. An extended range neutron rem counter. Nucl. Instrum. Meth. Phys. Res. A 297, 250-257.

Birattari, C., Dimovasili, E., Mitatoff, A., Silari, M., 2010. A Bonner sphere spectrometer with extended response matrix. Nucl. Instrum. Meth. Phys. Res. A 620, 260-269.

Esposito, A., Bedogni, R., Domingo, C., et al., 2010. Measurements of leakage spectra from high-energy accumulation ring using extended range Bonner sphere spectrometers. Radiat. Meas. 45, 1522-1525.

Galbiati, A., et al., 2009. Performance of monocrystalline diamond radiation detectors fabricated using TiW, Cr/Au and novel Ohmic DLC/Pt/Au electrical contact. IEEE Trans. Nucl. Sci. 56, 1863-1874.

ICRP, Publication 74, January 1997. Conversion Coefficients for Use in Radiological Protection against External Radiation, Annals of the ICRP. Elsevier Publ.

Marinelli, Marco, Milani, E., Prestopino, G., etal, 2006. High performance 6LiF-diamond thermal neutron detectors. Appl. Phys. Lett. 89, 143509. 1/4.

Martone, M., Angelone, M., Pillon, M., 1994. The $14 \mathrm{MeV}$ Frascati neutron generator. J. Nucl. Mater. 212-215, 1661-1664.

Pelowitz, D.B. (Ed.), 2005. MCNPXTM User's Manual, Version 2.5.0 Los Alamos National Lab. Report LA-CP-05-0369.

Pillon, M., Angelone, M., Krasilnikov, A.V., 1995. $14 \mathrm{MeV}$ neutron spectra measurements with $4 \%$ energy resolution using a type Ila diamond detector. Nucl. Instrum. Meth. Phys. Res. B 101, 473-483. 